\title{
El control de los antibióticos: ¿hasta donde duela?
}

\author{
ABEL JASOVICH ${ }^{*}$
}

\section{Control of antimicrobial agents: Control until it hurts?}

Utilizar un antibiótico (ATB) de manera racional significa relacionar correctamente el agente etiológico de la infección (inferido o documentado) con el fármaco que se elige. Dentro del espectro de racionalidad está la adecuación, es decir, la utilización del ATB más efectivo, con menos efectos adversos, por la vía de administración más fácil, y de menor costo.

La realidad es que la utilización inadecuada e irracional de los ATBs es frecuente en la práctica médica y constituye uno de los factores más importantes en la generación y la selección de resistencia bacteriana ${ }^{1,2}$. La magnitud del problema es tal que en el año 2000, The Pharmaceutical Research and Manufacturers of America (E.U.A.) comunicó que se encontraban en desarrollo 137 nuevos ATBs, 19 de ellos directamente focalizados en microorganismos multirresistentes ${ }^{3}$.

Como consecuencia de la infección por un patógeno resistente a múltiples ATBs, el paciente prolonga su estadía en el hospital, exige con frecuencia técnicas complejas de diagnóstico y, debido a que existen mayores posibilidades de falla terapéutica, requiere ATBs más caros y aumenta su riesgo de muerte ${ }^{4,5}$. Por lo tanto la resistencia bacteriana a los ATBs no debe ser considerada sólo en términos médicos sino también económicos.

En el ámbito hospitalario 23 a $38 \%$ de los pacientes recibe ATBs, en más del $50 \%$ de los casos de manera inapropiada ${ }^{6,7}$. La mayoría de estos casos corresponden a tratamientos empíricos realizados en instituciones donde documentar microbiológicamente las infecciones no es una práctica rutinaria. En las unidades quirúrgicas 40 a $50 \%$ de los pacientes recibe ATBs innecesariamente ${ }^{8}$. Asimismo, las profilaxis quirúrgicas son frecuentemente inapropiadas tanto en la elección del ATBs como en su duración?.
En este sentido, el desafío es implementar programas de uso de ATBs eficientes con alta probabilidad de éxito, y que al mismo tiempo aseguren la mejor calidad de la atención médica al menor costo.

Como el objetivo de estos programas es mejorar conductas médicas, en este caso particular el uso de ATBs, para que resulte más aceptable, el método a aplicar debe adaptarse a las características de la institución y al personal al cual va dirigido. Es decir, no existe un método referencial en el tema, y habitualmente es necesario combinar más de uno para aumentar las posibilidades de éxito. Ahora bien, al margen de la estrategia a utilizar, está demostrado que las acciones voluntaristas llevadas a cabo por personal no experimentado en el tema terminan en fracaso; por este motivo para que los programas de uso de ATBs resulten exitosos, deben ser conducidos y desarrollados por personal capacitado y en condiciones de liderazgo ${ }^{10}$.

\section{Políticas restrictivas}

La mayoría de las estrategias para el uso racional de ATBs se basan en políticas restrictivas tales como la indispensable autorización del infectólogo para el uso de ciertos antimicrobianos $^{11,12}$. La lista de ATBs restringidos suele estar conformada por los de precio elevado y en general no se la elabora con un criterio epidemiológico. Si bien con estas políticas se han reportado resultados exitosos en términos de reducción de resistencia bacteriana ${ }^{13}$ y mortali$\mathrm{dad}^{14}$, con ello pueden generarse situaciones conflictivas y no lograrse buena adhesión de los profesionales, quienes consideran que estas intervenciones lesionan su autonomía, característica primordial de la práctica médica ${ }^{15,16}$.

\footnotetext{
*Servicio de Infectología, Hospital Carlos Bocalandro. Buenos Aires, Argentina.

E-mail: ajasovich@jannsen.com.ar
} 


\section{Políticas no restrictivas}

En la literatura mundial son pocas las comunicaciones sobre experiencias exitosas de programas de control de ATB con libertad de prescripción.

Junto con mi grupo de trabajo, he comunicado una larga experiencia, documentada y validada sobre reducción de costos, consumo y mejoría de la calidad, resultante de la implementación de programas de optimización del uso de ATBs con libertad de prescripción. Esta metodología fue desarrollada en seis instituciones de diferente complejidad, tanto públicas como privadas ${ }^{17}(\mathrm{Ta}-$ bla 1); inclusive, en uno de los hospitales públicos en el cual este programa se implementó desde 1998, no hubo desabastecimiento de ATBs durante la profunda crisis económica que sufrió Argentina en el año 2002, y se pudo mantener la libertad de prescripción. En conclusión, un programa de optimización del uso de ATBs que funcionaba en el hospital previamente a la crisis con profesionales entrenados, permitió que todos los pacientes infectados recibieran el tratamiento antimicrobiano que tuvieran prescrito y que los indicadores de calidad como mortalidad cruda e infección de herida quirúrgica limpia se mantuvieran estables ${ }^{18}$. La metodología aplicada en todos los casos incluyó los siguientes componentes: acompañamiento del médico tratante hasta la resolución de la patología infecciosa del paciente, confección por consenso de un vademecum institucional, normatización de las profilaxis y de los tratamientos ATBs de patologías prevalentes, uso de formularios de pedido de ATB con fundamentación, discusión en ateneos institucionales para cambiar los hábitos prescriptivos, como el uso de antibióticos orales y devolución de los datos a los efectores como instrumento principal para el cambio de conductas.

Con este tipo de programas el médico puede prescribir el ATB que considere necesario sin restricciones, con el único requisito de fundamentar científicamente su pedido en un formulario diseñado para tal fin. La farmacia debe tener una postura férrea, es decir, no entregar el ATB si la orden de pedido no ha sido completada. Del análisis de la correlación entre el ATB solicitado, el fundamento del uso expresado por el profesional y las guías terapéuticas vigentes para cada patología, surgen los diferentes puntos en los cuales es necesario realizar una intervención correctora. El eje de este tipo de programas gira sobre el desplazamiento del liderazgo en el uso de ATBs hacia el infectólogo, es decir, lograr que el médico tratante prescriba ATBs con un criterio similar al del especialista líder ${ }^{10}$.

\section{Nuevas estrategias para optimizar el uso de antimicrobianos}

En la mayoría de las instituciones, las unidades hospitalarias destinadas a la asistencia de los pacientes críticos (UCI) constituyen las áreas de mayor utilización de ATBs y el principal reservorio de patógenos multirresistentes; es lógico entonces que las nuevas estrategias propuestas para optimizar el uso de ATBs estén focalizadas allí.

\section{Desintensificación del tratamiento antimi- crobiano (cobertura empírica máxima con re- ducción posterior)}

En varios estudios el tratamiento ATB tardío e inadecuado en pacientes críticos se asoció con un aumento significativo de la mortalidad ${ }^{19,20}$; asimismo, el excesivo uso de ATBs en UCI promueve la selección, emergencia y diseminación de microorganismos resistentes ${ }^{21,22}$ además de aumentar las posibilidades de superinfección en estos pacientes y los costos asociados. Priorizar una de estas dos conductas supone un dilema dífícil de resolver: maximización del resultado individual $v s$ minimización de la selección de microorganismos resistentes y del riesgo de superinfección.

La estrategia de desintensificación (deescalating therapy)del tratamiento ATB consiste en utilizar en pacientes graves la cobertura ATB empírica máxima hasta obtener resultados microbiológicos que permitan reducir el espectro de los antibacterianos utilizados. Para algunos autores, con esta metodología se reducen las posibilidades de que el tratamiento resulte inadecuado con un impacto mímimo sobre la epidemiología hospitalaria y sobre el riesgo del superinfección de paciente ${ }^{23,24,25}$. Sin embargo, esta postura se contrapone frecuentemente con la realidad de la práctica médica cotidiana: la mayoría de los médicos se resiste a reducir el espectro del ATB en situaciones en las que el paciente tiene una evolución favorable, sobre todo, aquellos profesionales en período de capacitación que son los mayores prescriptores en las UCI; la tasa de documentación microbiológica en los pacientes críticos es baja; en ocasiones, diferenciar contaminación de infección a partir de los cultivos positivos no es sencillo ni concluyente ${ }^{26}$. La mayoría de los estudios sobre desintensificación del tratamiento ATB con cobertura máxima inicial se realizaron en pacientes con neumonía asociada a ventilación mecánica (NAV); 


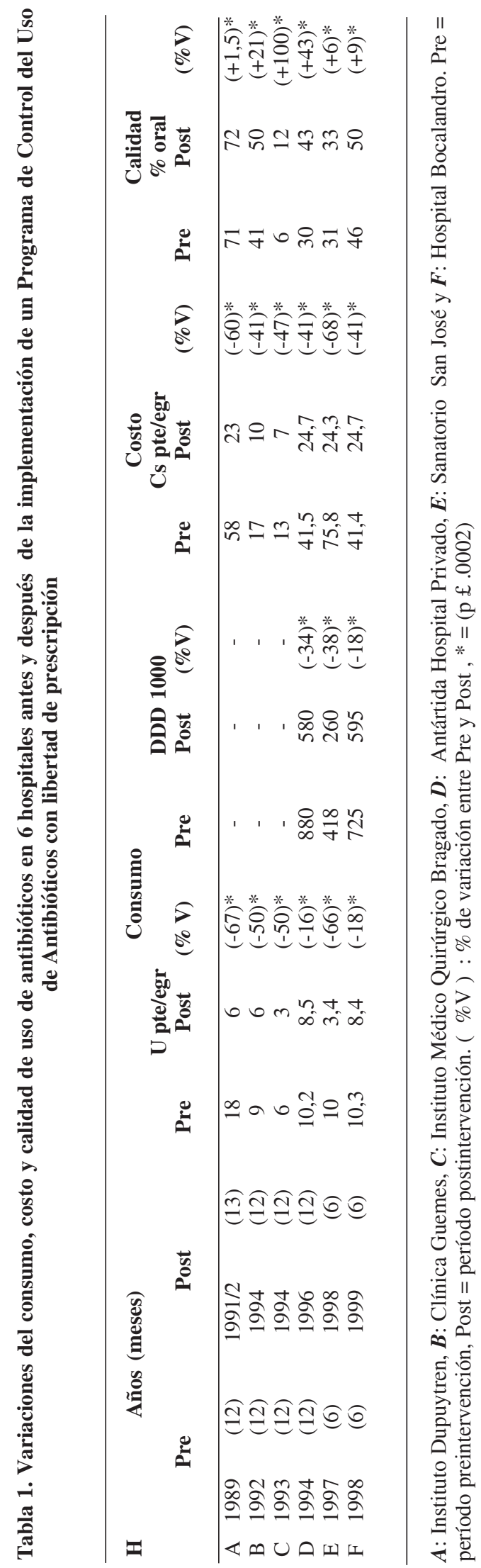


Tabla 2. Controversias en el diagnóstico de neumonía asociada a ventilación mecánica (NAV)

Subdiagnóstico

-Los datos clínicos, radiológicos y microbiológicos sólo hicieron diagnóstico de NAV en $62 \%$ de los casos*

Fagon J, Am J Med 1993; 94: 281-8

-Con la combinación de criterios clínicos y resultados de métodos invasores y no invasores de diagnóstico, el 15\% de las NAV no fueron diagnosticadas*

Fabregas N, Thorax 1999; 54: 867-73

Sobrediagnóstico
-Sólo $40 \%$ de los pacientes con clínica de NAV (fiebre, secreciones purulentas y nuevo infiltrado radiológico) tuvieron confirmación microbiológica. El $60 \%$ no recibió antibióticos y se recuperaron o en material de autopsia no se evidenció neumonía

Fagon J, Am Rev Respir Dis 1988;138:11

* comparado con el método referencial (cultivo de tejido pulmonar)

en esta patología, la sensibilidad y la especificidad de los criterios diagnósticos clínicos, radiológicos y microbiológicos son motivo de controversia permanente desde hace décadas (Tabla 2). Precisamente, Singh et al, analizaron el impacto clínico de la cobertura empírica máxima en pacientes con NAV, y demostraron que el tratamiento ATB excesivo tuvo un efecto negativo sobre la evolución de los pacientes ${ }^{21}$, en general, por errores en el diagnóstico inicial, por la prolongación excesiva de la terapia y por la indecisión del médico de reducir el espectro del tratamiento ATB. Por otra parte, en un estudio realizado en más de 900 pacientes internados en una UCI, Raymond et al, hallaron que la infección por bacilos Gram negativos multirresistentes (Pseudomonas aeruginosa, Acinetobacter spp y Stenotrophomona maltophilia) fue una variable predictora independiente de mortalidad, con lo cual el control de la diseminación de estos patógenos dentro de la UCI resultaría más efectivo que la utilización precoz de tratamientos empíricos de máxima cobertura ${ }^{27}$. Además, en este trabajo, fueron mejores los resultados clínicos en el grupo de pacientes en los que se realizó corrección del tratamiento ATB con los resultados microbiológicos. En síntesis, la aplicación de la estrategia de cobertura ATB empírica máxima requiere evaluar el costo-beneficio entre la necesidad de un tratamiento empírico inicial adecuado y el uso de ATBs potencialmente generadores de resistencia bacteriana y de complicaciones para el paciente y la institución; por tal motivo su utilización no puede generalizarse y debe restringirse sólo a un número reducido de pacientes cuya estratificación de riesgo lo justifique.
Cambios en los esquemas empíricos en respuesta a los brotes de patógenos resistentes

El fundamento de esta estrategia es evitar el uso empírico de determinados ATBs capaces de generar multirresistencia en determinados patógenos, particularmente en aquellas UCIs que se encuentran en situación de brote por alguno de estos microorganismos. El ejemplo clásico, ampliamente documentado en la literatura, lo constituyen las cefalosporinas de tercera generación. Monet et al, demostraron que el incremento en la utilización de ceftazidima en una UCI condicionó la aparición de Enterobacter cloacae resistente a este $\mathrm{ATB}^{22}$ Quale et al disminuyeron la prevalencia de pacientes colonizados con enterococo resistente a vancomicina mediante la adopción de una serie de medidas entre las que se destacaban la reducción en el uso de vancomicina, cefalosporinas y clindamicina ${ }^{28}$.

En series distintas Meyer et $\mathrm{al}^{29}$ y Peña et $\mathrm{al}^{30}$ comunicaron una significativa reducción en la tasa de resistencia a ceftazidima en Klebsiella pneumoniae al reemplazar este ATB por imipenem y piperacilina/tazobactam respectivamente.

Actualmente se está estudiando la influencia de otros ATBs como seleccionadores de patógenos resistentes al ser utilizados como tratamiento empírico en forma indiscriminada. Por ejemplo, las fluoroquinolonas pueden seleccionar ciertas mutantes de $P$. aeruginosa con sensibilidad disminuida a estos ATBs y aún con resistencia a los carbapenems como resultados del desarrollo de un mecanismo de impermeabilidad o de bomba activa de eflujo ${ }^{31}$. En un estudio de Carmeli et al la utilización de metronidazol parenteral fue un factor de riesgo independiente altamente significativo para la colonización por enterococo 
resistente a vancomicina ${ }^{32}$. Además, en otros estudios como el de Lautenbach et $\mathrm{al}^{33}$ se han observado resultados paradójicos como la asociación entre el uso de vancomicina y el aumento de infecciones hospitalarias por $E$. coli y $K$. pneumoniae productoras de $\beta$-lactamasas de espectro extendido. Recientemente, Bantar et al han comunicado una reducción en las tasas de resistencia de Proteus mirabilis y E. cloacae a partir de la variación en las proporciones de uso de determinados ATBs con respecto de las cefalosporinas de tercera generación. Cefepima y aminopenicilinas con inhibidores de $\beta$ lactamasas son dos ejemplos. El aumento de este último ATB correlacionó con una disminución de la tasa de resistencia de Staphylococcus aureus a meticilina $^{34}$.

Algunos autores recomiendan que el hospital cuente con un vademecum de ATB amplio, para evitar que el uso reiterado de un grupo reducido de antibacterianos ejerza presión de selección sobre las bacterias nosocomiales.

\section{Ciclado de antimicrobianos}

El ciclado consiste en seleccionar un ATB en particular o un grupo de ellos y no utilizarlos por un período de tiempo determinado en el cual es sustituido por otro ATB aprobado para las mismas indicaciones. Habitualmente los períodos de ciclado van de 1 mes a 2 años ${ }^{35}$. En teoría esta estrategia promueve la reducción de los niveles de resistencia al ATB reemplazado, lo que permitiría volver a utilizarlo con relativa seguridad una vez finalizado el período de ciclado; es decir, los cambios en el uso de ATB son seguidos por cambios en la resistencia bacteriana.

Sin embargo, uno de los primeros estudios sobre ciclado de ATB tuvo resultados controversiales. Domínguez et al observaron una reducción de las tasas de resistencia en bacilos Gram negativos de su UCI cuando compararon cuatro períodos de tiempo en cada uno de los cuales se utilizaron diferentes tipos de ATBs, pero también documentaron un incremento en la tasa de resistencia de cocos Gram positivos, básicamente Enterococcus $\mathrm{sp}^{36}$. Por el contrario, John et al, implementaron un ciclado cuatrimestral de ATB en los tratamientos empíricos de una UCI quirúrgica y reportaron reducciones de la mortalidad atribuible a enfermedades infecciosas y de las tasas de resistencia bacteriana en gene$\mathrm{ral}^{37}$. Otro estudio de ciclado cuatrimestral fue desarrollado por Raymond et $\mathrm{al}^{38}$; los ciclos de ATBs incluyeron el uso de ciprofloxacina, imipenem, piperacilina-tazobactam y cefepima. La incidencia de infección por bacterias Gram positivas y Gram negativas multirresistentes, así como la mortalidad atribuible a enfermedades infecciosas fue significativamente menor en el período de ciclado que en el año precedente.

Aunque la estrategia impresione como simple, en realidad es compleja, debido a que no son sencillas de definir las variables implicadas: ATB a ciclar, ATB de reemplazo, tiempo de ciclado y área del hospital donde realizarlo. La prevalencia institucional de determinados patógenos ( $S$. aureus resistente a meticilina, bacilos Gram negativos productores de $\beta$-lactamasas de espectro extendido, bacilos Gram negativos no fermentadores) puede condicionar la selección del ATB a ciclar y su reemplazo. Al respecto, Bochorishvili et al $^{39}$ comunicaron que $50 \%$ de los pacientes tratados con ATB requirieron antibacterianos que estaban fuera del proceso de ciclado. Estos y otros aspectos inherentes a la implementación conspiran contra la confiabilidad y la aceptación de esta metodología. El desarrollo futuro de trabajos de investigación correctamente diseñados y con un número importante de pacientes, permitirá medir el verdadero valor de esta atractiva estrategia de control del uso de ATBs.

\section{Conclusiones}

Luego de haber desarrollado las diferentes estrategias propuestas, la pregunta es: ¿es necesario avanzar en el control del uso de ATB hasta que duela? Poco sustento tendría cualquier respuesta si primero no identificamos correctamente el objeto de tamaño sufrimiento: ¿hasta que le duela al médico prescriptor? (restringiéndole el uso de determinados ATB o modificando su prescripción por el infectólogo) ¿a las bacterias que causan las infecciones? (cobertura empírica máxima), ¿a la flora hospitalaria? (cambios de los tratamientos empíricos en respuesta a los brotes). Lo cierto es que independientemente del método que se utilice, suele haber grandes diferencias en la densidad de uso de ATBs entre hospitales con iguales características e igual tipo de pacientes internados. Esta variabilidad sugiere que el uso de ATBs en los centros asistenciales tiene mucha relación con el liderazgo de ciertos profesionales y su hábito particular de prescripción ${ }^{40}$. En este sentido, en una institución privada de alta complejidad con 200 camas de internación hemos demostrado (AJ et al) que utilizando como única estrategia el liderazgo del infectólogo acompañando al médico de cabecera, se redujo significativamente el consumo de vancomicina, cefalosporinas de tercera generación y meropenem con aumento del uso de ampicilina-sulbactam y 

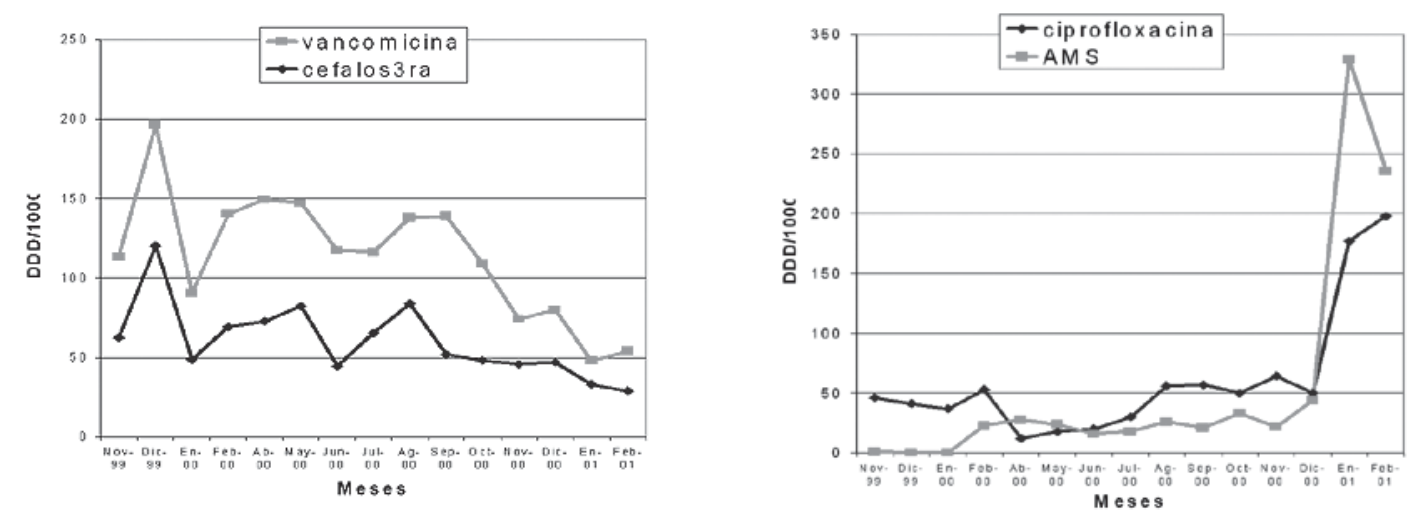

Figura 1. Consumo de antimicrobianos seleccionados. Variaciones observadas con un programa con libertad de prescripción (dosis diaria definida por 1.000 pacientes/día -DDD/1.000-).

ciprofloxacina especialmente en sus formulaciones orales (Figura 1) ${ }^{41}$. Además, contra la creencia de que es necesario utilizar métodos restrictivos para obtener resultados rápidos, en este trabajo demostramos que el desplazamiento del liderazgo en el uso de ATBs hacia el infectólogo se logró tan rápidamente que a los 30 días de iniciado el programa se obtuvieron resultados favorables. En una encuesta realizada en la misma institución la mayoría de los profesionales manifestaron sentirse cómodos con este tipo de metodología y que ella no afectaba su autonomía (datos propios no publicados).

En conclusión, existe la posibilidad de actuar sobre las conductas de uso de ATBs y mejorarlas, pero resulta indispensable considerar el contexto en forma global: la prescripción de un determinado fármaco es un acto médico cuya fundamento supera cualquier método restrictivo, guía terapeútica o proceso de desintensificación o ciclado. Sólo con un convencimiento firme es posible cambiar el hábito prescriptivo, de allí que la presencia y el trabajo cotidiano con un profesional con experiencia e idoneidad reconocida en el tema de ATBs, respetado por sus pares como tal, es indispensable para lograr resultados exitosos $^{34,42}$.

\section{Bibliografía}

1.- Goldmann D A, Weinstein R A, Wenzel R P, et al. Strategies to prevent and control the emergence and spread of antimicrobial-resistant microorganisms in hospitals: A challenge to hospital leadership. The Workshop to Prevent and Control the Emergence and Spread of Antimicrobial-Resistant Microorganisms in Hospitals. JAMA 1996; 275: 234-40

2.- Polk R. Optimal use of modern antibiotics: Emerging trends. Clin Infect Dis 1999; 29: 264-74.

3.- Pharmaceutical companies are working on 137 new ways to thwart infectious diseases, says PhRMA survey. Available at: http://www.phrma.org/press/newsreleases/ 2000-10-31.182.phtml. Accessed July 30, 2001

4.- Kereselidze T, Maglacas A M. Nosocomial Infections-what WHO is doing. J Hosp Infect 1984; 5(suppl A): 7-11.

5.- Acar J F. Consequences of bacterial resistance to antibiotics in medical practice. Clin Infect Dis 1997; 24 (Suppl 1): S17-8.

6.- Maki D G, Schuna A A. A study of antimicrobial missuse in a university hospital. Am J Med Sci 1978; 275: 271-82.

7.- Cooke D M, Salter A J, Phillips I. Antimicrobial missuse, antibiotic policies and information resources. J Antimicrob Chemother 1980; 6: 435-43.

8.- Scheckler W E, Bennett J V. Antibiotic use in seven community hospitals. JAMA 1970; 213: 264-7.

9.- Clausen D C, Evans R S, Petronik S L, et al. The timing of prophylactic antibiotics and the risk of surgical wound infection. N Engl J Med 1992; 326: 281-6.

10.- Thomsom O’Brien M, Oxman A, Haynes R et al. Local opinion leaders: effects on professional practice and health care outcomes. Cochrane Database Syst Rev 2000; (2): CD000125.

11.- Nathwani D. How do you measure the impact of an antibiotic policy? J Hosp Infect 1999 Dec; 43 Suppl: S265-8.

12.- Crowe H M, Quintiliani R. Antibiotic Formulary Selection. In: Cunha B, Guest Editor The Medical Clinics of North America, Antimicrobial Therapy I, W.B. Saunders Company May 1995: 79 (3); 463-76.

13.- Paterson D L, Restrictive antibiotic policies are appropriate in intensive care units. Crit Care Med 2003; 31 (1).

14.- Du B, Chen D, Liu D et al. Restriction of thirdgeneration cephalosporin use decreases infection-related mortality Crit Care Med 2003; 31 (4).

15.- Kassirer JP. Doctor discontent. N Engl J Med 1998; 339 (21):1543-4.

16.- Mainous A G. Controlling antibiotic resistance: Will we someday see limited prescribing autonomy? Amer Fam Phys 2001; 63(6).

17.- Jasovich A, Curcio D, Prieto S et al. Is it feasible to reduce consumption and cost of antibiotics with 
freedom of prescription ? A 10-year experience in 6 hospitals. Abstract of the III Panamerican Congress of Infection Control and Hospital Epidemiology. Belo Horizonte, Brazil, Nov 2000.

18.- Jasovich A, Curcio D, Alejandre S et al. Respuesta ante la crisis: Contribución de un Programa de Optimización del Uso de Antibióticos al funcionamiento de un hospital con recursos limitados (Abstract 94). En Libro del Resúmenes del XI Congreso de la Asociación Panamericana de Infectología Córdoba, Argentina 2003.

19.- Kollef M H, Sherman G, Ward S, et al. Inadequate antimicrobial treatment of infections: A risk factor for hospital mortality among critically ill patients. Chest 1999; 115: 462-74.

20.- Ibrahim E H, Sherman G, Ward S et al. The influence of inadequate antimicrobial treatment of bloodstream infections on patient outcomes in ICU setting. Chest 2000; 118: 146-55.

21.- Singh N, Rogers P, Atwood C W, Wagener M M, Yu V L. Short course empiric antibiotic therapy for patients with pulmonary infiltrates in the intensive care unit: A proposed solution for indiscriminate antibiotic prescription . Am J Respir Crit Care Med 2000 Aug; 162: 505-11

22.- Monnet D, Archibald L, Phillips L, et al. Antimicrobial use and resistance in eight US hospitals: complexities of analysis and modeling. Infect Control Hosp Epidemiol 1998 June; 19(6): 388-94.

23.- Höffken G, Niederman M S. Nosocomial pneumonia The importance of a De-escalating strategy for antibiotic treatment of pneumonia in the ICU. Chest 2002 Dec; 122 (6): 2183-96. Review.

24.- Niederman MS. An approach to empiric therapy of nosocomial pneumonia. Med Clin North Am 1994; 78: $1123-41$.

25.- Luna $C$, Vujacich $P$, Niederman $M$ et al. Impact of BAL data on the therapy and outcome of ventilatorassociated pneumonia. Chest 1997; 111: 676-85.

26.- Paterson D, Rice L. Empirical antibiotic choice for the seriously ill patient: are the minimization of selection of resistant organisms and maximization of individual outcome mutually exclusive? Clin Infect Dis 2003; 36:1006-12.

27.- Raymond D, Pelletier S, Cractree T et al. Impact of antibiotic-resistant Gram-negative bacilli infections on outcome in hospitalized patients. Crit Care Med 2003; 31(4).

28.- Quale J, Landman D, Saurina G et al. Manipulation of a hospital antimicrobial formulary to control an outbreak of vancomycin-resistant enterococci. Clin Infect Dis 1996; 23: 1020-5.

29.- Meyers K S, Urban C, Eagan J A et al. Nosocomial outbreak of Klebsiella infection resistant to lategeneration cephalosporins. Ann Intern Med 1993; 119: 353-8.

30.- Peña C, Pujol M, Ardanuy $C$ et al. Epidemiology and successful control of a large outbreak due Klebsiella pneumoniae producing extended-spectrum $\beta$-lactamases. Antimicrob Agents Chemother 1998; 42: 53-8.

31.- Livermore D M. Multiple mechanisms of antimicrobial resistance in Pseudomonas aeruginosa: Our worst nightmare? Clin Infect Dis 2002; 34: 634-40.

32.- Carmeli Y, Eliopoulos G M, Samore M H: Antecedent treatment with different antibiotic agents as a risk factor for vancomycin-resistant Enterococcus. Emerg Infect Dis 2002; 8: 802-7.

33.- Lautenbach E, Baldus Patel J, Bilker W et al. Extended-spectrum $\beta$-lactamase-producing E.coli and $K$. pneumoniae: Risk factors for infection and impact of resistance on outcomes. Clin Infect Dis 2001; 32: 1162-71.

34.- Bantar C, Sartori B, Vesco E et al. A hospitalwide intervention program to optimize the quality of antibiotic use: Impact on prescribing practice, antibiotic consumption, cost savings, and bacterial resistance. Clin Infect Dis 2003; 37: 180-6.

35.- Lavin B. Antibiotic cycling and marketing into the 21st century: A perspective from the pharmaceutical industry. Infect Control Hosp Epidemiol 2000; 21 (Suppl): S32-S35.

36.- Domínguez E A, Smith T L, Reed E et al. A pilot study of antibiotic cycling in a hematology-oncology unit. Infect Control Hosp Epidemiol 2000: 21:S4-S8.

37.- John J F Jr, Rice L B: The microbial genetics of antibiotic cycling. Infect Control Hosp Epidemiol 2000; 21 (Suppl): S22-S31.

38.- Raymond D, Pelletier D, Crabtree T et al. Impact of a rotating empiric antibiotic schedule on infectious mortality in an intensive care unit. Crit Care Med 2001; 29: 1101-8.

39.- Bochorishvili V, Madariaga M, Pur S et al. Reasons for non-compliance with voluntary cycling of antibiotics (abstract 109) In: Programs and Abstracts of de $40^{\text {th }}$ Annual Meeting of the Infectious Diseases Society of America 2002: 64.

40.- Fridkin S, Edwards J, Courval J et al. The effect of vancomycin and third-generation cephalosporins on prevalence of vancomycin-resistant enterococci in 126 US adult intensive care units. Intensive Care Antimicrobial Resistance Epidemiology (ICARE) Project and the National Nosocomial Surveillance (NNIS) System Hospitals. Ann Intern Med 2001; 135: 175-83.

41.- Jasovich A, Curcio D, Caso Nuñez H et al. Impact of the infectious disease team on the rationale for antibiotic usage: an expeditious intervention strategy avoiding the physician freedom limitation. J Chemother 2003; 15(4): 348-50.

42.- John J F Jr, Fishman N O. Programmatic role of the infectious diseases physician in controlling antimicrobial costs in the hospital. Clin Infect Dis 1997; 24 (3): 471-85. 